\title{
MEDICAL PRACTICE
}

\section{Hospital Topics}

\section{Bright's Disease Today: The Pathogenesis and Treatment of Glomerulonephritis*-I}

\section{J. S. CAMERON}

British Medical fournal, 1972, 4, 87-90

"There is nothing final nor absolute in the description of disease. . . Pathology cannot reach beyond the limits of knowledge in the basic sciences, and current ideas cannot escape the scientific climate of the time, nor exceed the bounds imposed by the immediate rule of philosophy or even of language."-W. N. MANN. ${ }^{1}$

\section{Summary}

Since Bright's day the study of glomerulonephritis has largely consisted in the observation of the clinical, biochemical, and morphological characteristics of patients. Linked to this has been the attempt to name and classify these data in a fashion which improved prediction of outcome and response to treatment. This has proved difficult, since there is no absolute correspondence between clinical syndromes and the morphological findings in the kidney. The histological data have, however, allowed good prediction of prognosis and response.

We now have enough information from experimental models of nephritis and from clinical observation to outline the pathogenesis of glomerulonephritis. Immune mechanisms are certainly concerned in some patients, the role of chronic soluble complex deposition in the kidney being particulary important. Non-immunological origins and mediation of injury, however, are at least as important. Injury to the glomerulus is mediated through the interaction of complement and coagulation mechanisms, leading through the release of soluble factors to inflammation. Repair is probably central in producing irreversible glomerular damage.

Treatment can now be directed towards the events concerned in the pathogenesis of nephritis, the most accessible at

* Based on the Goulstonian lecture delivered at the Royal College of Physicians of London on 14 March 1972

Guy's Hospital, London S.E.1

J. S. CAMERON, M.D., F.R.C.P., Consultant Physician in Renal Diseases and Senior Lecturer in Medicine the moment being the immediate mediators of injury. At a more fundamental level better identification in individual patients of the events leading to the injury is needed, such as the isolation, avoidance, or elimination of the antigens involved in chronic soluble complex disease. Laboratory techniques to begin these investigations in man are now available and need to be applied more widely in the clinical field to patients with glomerulonephritis.

\section{Early Theories}

In 1833 Richard Bright gave the Goulstonian lectures. The result was the middle paper in a triad of classic publications which defined the relation between dropsy, coagulable urine, and renal disease. ${ }^{2-4}$ The Goulstonian lecturers were particularly notable for including the first description of a rising blood urea with advancing renal impairment. The full description of the clinical and gross morbid anatomical features of all stages of glomerulonephritis in Bright's paper of $1836^{4}$ is justly regarded as one of the greatest of medical classics.

In his book of $1827^{2}$ and address in 1833 , however, he considered only chronic disorders with a fatal outcome. These were characterized clinically by proteinuria, oedema, and uraemia. Nowadays we would call these examples of the nephrotic syndrome leading on to chronic renal failure and hypertension. It then became apparent from work in Paris and Edinburgh that acute postscarlatinal nephritis could also be regarded as a variety of Bright's disease. By 1836 Bright supposed that all the chronic renal disease he saw had gone through an initial hyperaemic phase which might or might not be clinically visible. This was an attitude which persisted, numbering among its more recent advocates Thomas Addis.

The contrary view, that acute hyperaemic illnesses of the kidney might represent altogether distinct disorders, was also current in Bright's time and was most recently expounded by Longcope and Ellis. Bright did not see sick children, nor did he recognize that simple albuminuria could persist for years 
without renal damage. ${ }^{5}$ Most of the more benign clinical forms of glomerulonephritis, including the nephrotic syndrome of childhood, recurrent haematuria, and isolated proteinuria, did not therefore come to his attention. His picture was of necessity incomplete.

Now we realize that the situation is even more complex than Bright and his immediate successors supposed. Mann ${ }^{1}$ reviewed very well the gradual evolution of ideas on the clinical and morbid anatomical features of Bright's disease. The principal advances in the nineteenth century were the separation of "essential" hypertension as a primary cause of contracted kidneys, the recognition of the gross and minute picture of chronic pyelonephritis, and the realization that systemic disorders, such as amyloidosis and diabetes, could mimic the syndromes of Bright's disease by affecting the kidney.

\section{Modern Observations}

The most recent attempt to provide a simple clinicopathological classification of nephritis was that of Ellis in his Croonian Lectures of $1942 .^{6}$ While his classification holds clinically, it fails badly when clinical presentation and histological appearances are put together. The damaged kidney can express injury at a clinical level through only a limited number of syndromes, which are varying combinations of haematuria, proteinuria and its consequences, and loss of functioning nephrons. Similarly, at the morphological level only a limited number of patterns of structural damage can be discerned. I have dealt with these in detail elsewhere, ${ }^{78}$ and they were the subject of a recent conference. ${ }^{9}$ I shall not discuss them further here. When these clinical syndromes and histological appearances are correlated, as in the study of our last 500 biopsied patients with primary glomerular disorders (see Table), there is some correspondence, as Ellis sugges-

Modes of Presentation and Histological Appearances in Primary Glomerular Disease

\begin{tabular}{|c|c|c|c|c|c|c|c|}
\hline \multirow{2}{*}{\multicolumn{3}{|c|}{ Histological Finding }} & \multicolumn{4}{|c|}{ Presentation } & \multirow{2}{*}{ Total } \\
\hline & & & A.G.N. & R.H. & P.P. & N.S. & \\
\hline 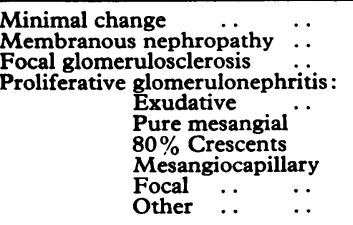 & $\begin{array}{l}\ldots \\
\cdots \\
\cdots \\
\cdots \\
\cdots \\
\cdots\end{array}$ & $\begin{array}{l}\because \\
\because \\
\therefore \\
\because \\
\because \\
\because \\
\therefore\end{array}$ & $\begin{array}{c}0 \\
0 \\
0 \\
9 * \\
18^{*} \\
15^{*} \\
18 \\
0 \\
8\end{array}$ & $\begin{array}{l}7 \\
0 \\
1 \\
0 \\
4 \\
0 \\
5 \\
2 \\
9\end{array}$ & $\begin{array}{r}18 \\
11 \\
11 \\
1 \\
5 \\
0 \\
17 \\
4 \\
36\end{array}$ & $\begin{array}{r}164^{*} \\
30^{*} \\
22^{*} \\
3 \\
21 \\
4 \\
30 \\
5 \\
48\end{array}$ & $\begin{array}{r}189 \\
41 \\
34 \\
13 \\
48 \\
19 \\
70 \\
11 \\
101\end{array}$ \\
\hline & & tal & 68 & 28 & 103 & 327 & 526 \\
\hline
\end{tabular}

*These figures include a total of 28 patients who presented in acute renal failure and

required dialysis.
A.G.N. = Acute glomerulonephritis. R.H. = Recurrent haematuria. P.P. = Persistent symptomless haematuria. N.S. = Nephrotic syndrome.

ted. But even with the refinements of morphology brought about by technical advances since Ellis's days, such as electron microscopy and renal biopsy, this is far too poor to predict any one histological appearance from a clinical presentation or the clinical picture from the histological findings.

The importance of identifying the patterns of structural damage seen on renal biopsy (see Table) is that unlike the clinical syndromes, which have a quite variable prognosis, the histological data provide an excellent but gloomy guide to the outcome the patient and doctor may expect. ${ }^{10}$ A life table analysis of patients with the various appearances which can be distinguished microscopically is shown in Fig. 1. The patients fall into three groups. Death is unusual in patients with normal or nearly normal glomeruli, acute exudative lesions, proliferation and sclerosis confined to the mesangium, and focal proliferative disorders without systemic vas-

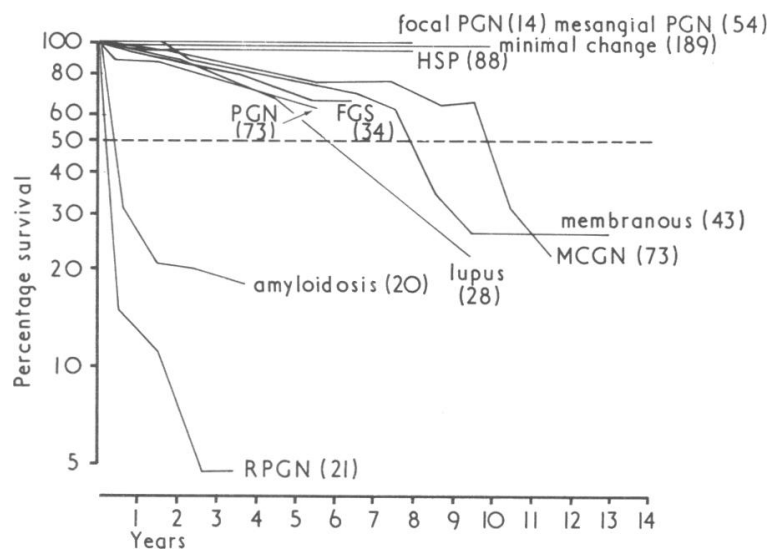

FIG. 1-Actuarial survival44 of patients followed at Guy's Hospital and suffering from glomerular disease. The allocations are by histological appearances on renal biopsy, with the exception of lupus nephritis nad enoch-Schönlein purpura.

FGS = Focal glomerulosclerosis. MCGN=Mesangiocapillary (membranoproliferative) glomerulonephritis. RPGN = Proliferative glomerulonephritis with more than $80 \%$ of glomeruli affected by large epithelial crescents (rapidly progressive glomerulonephritis on clinical classification). PGN =Other proliferative glomerulonephritis, after the other groups specified in the figure are analysed separately. HSP $=$ HenochSchönlein purpura

The histological terms are defined in detail elsewhere. 7810

culitis. In contrast, there is a steady mortality in patients with patterns of obvious structural damage with scarring, such as membranous nephropathy, focal glomerulosclerosis, proliferative glomerulonephritis, and mesangiocapillary (membranoproliferative) glomerulonephritis. Finally, patients with extensive crescent formation as well as glomerular proliferation do very badly indeed.

The present clinical importance of identifying these patterns of structural damage and the work that has gone into their identification from many units (including our own) over the past decades should not delude us into thinking of these changes as diseases in their own right. They represent responses which almost certainly result in each case from a variety of stimuli. Conversely, as I shall illustrate below, the same basic pathological processes can lead to a great variety of patterns of structural damage.

The application of clinical biochemistry and pharmacology has improved our management of patients, and the more minute analysis of histological appearances has improved our ability to give a prognosis. The recognition of the patterns of focal glomerulosclerosis ${ }^{11}$ and mesangiocapillary glomerulonephritis $^{12}$ are recent examples but represent essentially modern applications of the techniques of clinical observation and investigation available to Bright. The unravelling of glomerulonephritis is now, however, predominantly a problem for experimental and clinical pathology, and not of morbid histology. If we are to improve our treatment of these disorders, which are an important cause of death in young adults, ${ }^{13}$ we must consider them at a more fundamental level than at either the morphological or clinical level.

Although most of our own work in the past decade has been in these areas of clinical observation and morbid histology as a necessary step in progress, I shall spend the greater part of this lecture in reviewing what is now known of the pathogenesis of glomerular disease and how glomerular injury is produced. On this basis I shall try to relate the information to present and possible future therapy.

\section{Origins of Glomerulonephritis}

What do we know of the events that lie behind the clinical features and morphological observations of glomerulonephritis? As so often happens in medicine we are faced with an unwieldly body evidence, some of it clinical and some experimental, heavily orientated not by the questions we would 
like to ask but by the techniques capable at present of providing answers.

The bulk of clinical and experimental evidence suggests that mechanisms involved in immunity and inflammation are also involved in glomerulonephritis. Beyond this rather bland statement it is very difficult to be precise. The evidence stems from two broad sets of observations. The first is that diseases looking remarkably like human glomerulonephritis, both clinically and morphologically, can be produced in a number of animal species by manipulation of immune mechanisms. The second is that in man an important minority of clinical conditions appear to relate to acute or chronic infection and to defined alterations in immune systems.

Immunology is a fashionable and rapidly expanding area of experimental pathology at the moment, and we should perhaps be cautious and not allow our growing expertise in this field to blind us to the fact that the range of response of both tissues in general and the kidney in particular is very limited; they trot out the same response whatever stimulus is applied. The similarities between some of the experimental conditions and disease in man may reflect more this poverty of response than any fundamental similarity in pathogenesis. Nonimmunological mechanisms, especially those involving surface damage and coagulation, are clearly important and likely to appear more so in the future. The first line of Fig. 2 emphasizes this.

\section{PRECIPITATING FACTORS}

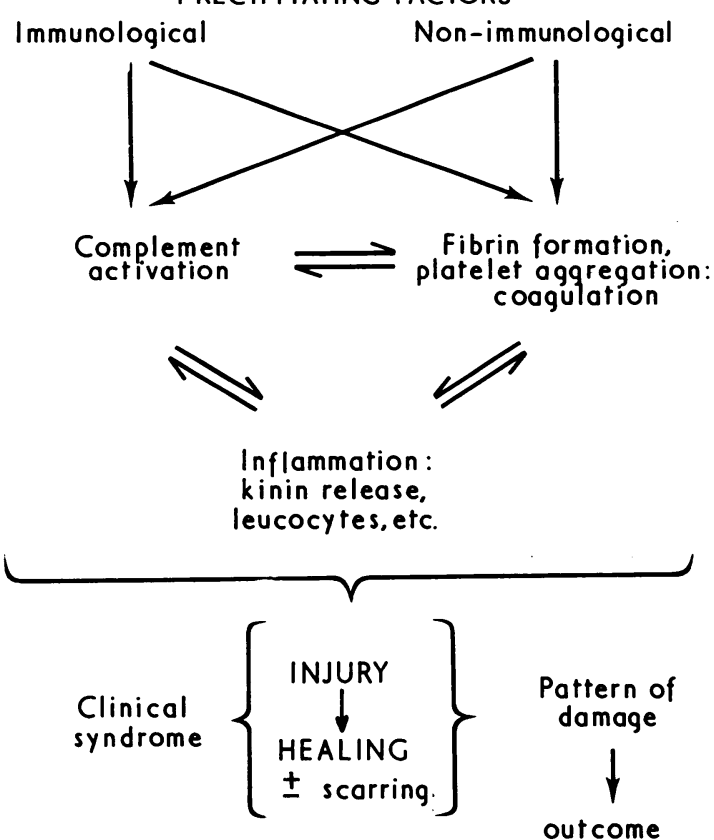

FIG. 2-Levels of descriptive diagnosis in glomerulonephritis. Most current description is at the level of patterns of structural damage, which seem to influence outcome (Fig. 1), and the presenting syndrome, which does not. Complete description of each patient requires, in addition to glomerular morphology and clinical observation, a statement of the initiating circumstances and route of mediation of injury. Since this may be modified by many factors at all levels shown in the diagram a simple one-to-one relation between causes and effects cannot be anticipated except in the broadest sense.

Present evidence suggests that of the two arms of the immune response, cell-mediated and humoral-antibodymediated immunity, it is the antibody-mediated responses that are of the greater importance. The only exception to this appears to be homograft rejection in non-immunized, immunosuppressed recipients where the kidney is rejected mainly by cell-mediated mechanisms." Again it is unlikely that any stimulus is followed by a response exclusively through antibody-mediated or cell-mediated pathways. In any case, both involve cells in the first place for recognition of antigen and production of antibody, so that the division is to some extent artificial. ${ }^{15-18}$ With these warnings in mind we can now examine the position with regard to experimental nephritis in animals and clinical nephritis in man.

Two main types of antibody-mediated assault on the kidney can be discerned ${ }^{19-20}$ - (1) antibody directed against tissuefixed antigen, in this case kidney; and (2) antibody combined with circulating antibody to form antigen-antibody complexes. In the second case the antigen is not specific and may be exogenous-for example, viral or bacterial-or endogenous. To complicate matters still further, antibody against viral or bacterial antigen may cross-react with tissue antigens.

A great deal of experimental work indicates that the presentation of antigen is not followed by a simple predictable response in all the recipients, whether animals or man. In modulating the final response the following factors seem to be important. (1) Genetic factors-(a) the variation in inherited ability to mount an antibody response (variables here are the types of antibody, duration of the response, quantity of antibody); (b) the balance between cell-mediated and humoral antibody responses evoked by antigens ( $B$ and $T$ lymphocyte response). (2) The antigen-nature, route and mode of presentation, quantity, and duration of exposure. (3) The presence of further modifying factors, such as coincident infection or inflammation.

Obviously we cannot expect the induction and possible progression of immune nephritis to be a simple affair if all these variables are taken into consideration. Firstly, let us look at the situation of soluble complex nephritis, because it is probably of greatest relevance to chronic renal disease in man.

\section{Soluble-complex-induced Glomerulonephritis}

Von Pirquet ${ }^{21} 22$ noted that the kidney was one of the organs affected during acute serum sickness in man, along with joints and the heart. He postulated that a "toxic body" was formed during a phase when both antigen and antibody coexisted in the plasma. Longcope ${ }^{23}$ and McClean et al. ${ }^{24}$ showed that a chronic disease could be produced by repeated immunizations. Dixon and his colleagues explored this model systematically. $1925-27$ Their work is well known and I shall only summarize it briefly here.

Firstly they studied "one-shot" serum sickness nephritis in rabbits and showed that the appearance of lesions in the heart, kidneys, and elsewhere followed immediately on the formation of somewhat small circulating soluble complexes, rather than the usual very large insoluble complexes removed by phagocytosis or by the reticuloendothelial system. These soluble complexes are Von Pirquet's "toxic body." Extensive studies of these toxic complexes have shown that they are formed in slight antigen excess in animals which are rather poor at forming antibody. ${ }^{28}$ The glomerulus receives one-fifth of the cardiac output and has a very permeable capillary circulation compared with those elsewhere in the body, and a large proportion of complexes are filtered off in the glomerular capillary circulation and become visible as "humps" of material in an extracapillary situation. In these granular deposits immunofluorescent staining techniques can demonstrate antigen, antibody, and complement components. The antigen in this model is quite unrelated, therefore, to the kidney. In many experimental models it has been heterologous serum albumin.

In rabbits it has been possible to "titrate" the animal with just enough antigen to preserve slight antigen excess over a long period. ${ }^{19} 20$ Then, instead of developing an acute selflimiting disease the animals suffer from a chronic nephritis which may resemble histologically membranous nephropathy or proliferative glomerulonephritis. Some animals which are rather poor antibody formers are therefore particularly prone to situations of antigen excess in chronic infections. Soothill and Steward $\mathrm{d}^{30}$ also showed that mice of nephritis-prone strains 
produce antibody of lower affinity than nephritis-resistant strains when exposed to antigens. Thus both the quantity and the quality of antibody are important.

The possible role of chronic virus infection in the induction of chronic nephritis in animals has been extensively studied. ${ }^{31}$ Several species show naturally occurring disease, such as the Aleutian disease in mink, ${ }^{32}$ or experimental conditions can be induced by chronic viral infections. The best studied species is the mouse. In mice a variety of viruses may cause nephritis, such as the lymphocytic choriomeningitis virus. ${ }^{31}$ Viruses are especially apt to produce chronic antigen load since they are intracellular, long lived, and self-replicating.

Another important model of experimental nephritis is the renal disease developing in the crossed New Zealand Black/ New Zealand White mice. ${ }^{33}$ These animals develop a condition which has many resemblances to human systemic lupus erythematosus, including a glomerulonephritis responsible for the death of most of the animals. This nephritis seems to be initiated by DNA and anti-DNA soluble complexes. Talal and his colleagues ${ }^{34} 35$ obtained evidence that these mice have an inherited imbalance in their immune responses consisting of excessive antibody response to administered antigens at the same time as their $\mathrm{T}$ lymphocytes are resistant to the induction of tolerance.

Elution of the antibody from the glomeruli of these mice, presumed to be part of antigen-antibody complexes, showed that although $50 \%$ of the antibody was anti-DNA antibody, as expected, a major proportion of the other $50 \%$ reacted with the endemic Gross virus, which in normal mice does not evoke an antibody response. ${ }^{36}$ The presence of the Gross virus in the mice, as with coincidental infection with polyoma and lymphocytic choriomeningitis viruses, appeared to enhance the appearance of anti-DNA antibody and renal disease. Even more interesting, however, was the observation that infection with the lactic dehydrogenase virus protected the animals against nephritis and anti-DNA antibody. Chronic virus infection in these animals can therefore modulate the disease arising in the setting of an inherited imbalance of the immune system. The implications of this work for human glomerulonephritis are considerable.

Even after considering all the variables that may determine whether potentially toxic soluble complexes appear in the circulation, there are a number of factors which may allow or deny them lodgement to the vessel or capillary walls. ${ }^{37}$ Simple filtration will permit the rather small toxic complexes to penetrate glomerular capillaries to some extent, but this is greatly increased if there is coincident inflammation and increased vascular permeability. This is the inevitable result of complement activation, which I shall discuss later, but it appears that in the acute soluble complex disease in rabbits injury can take place in the absence of complement. ${ }^{38}$ Cochrane $^{37}$ described in rabbits a cell-mediated pathway of increased capillary permeability involving IgE, basophil leucocytes, platelets, and the liberation of histamine, serotonin, and vasoactive amines. This leads to the localization of the otherwise non-toxic circulating complexes and consequent injury.

Finally, when soluble complexes are deposited within the glomerulus they generally become undetectable after a few weeks. The exact route of removal is not clear, but almost certainly the mesangial cells behave in some respects as reticuloendothelial cells and play a major part in the removal of complexes. Variations in the rate of this removal could be critical in the induction of disease.

\section{Anti-kidney Antibody (Nephrotoxic Serum Nephritis)}

This variety of glomerulonephritis was the earliest experimental model ${ }^{39}$ and was extensively studied by Masugi and others. In various forms it has provided many data. ${ }^{26}$ In this model the antibody is specifically directed against renal tissue. When an antibody raised in a foreign species to kidney extracts is reinjected into the original animal ${ }^{40}$ combination of antibody with antigen in the glomerular capillary walls takes place. Another variation of greater relevance to man is the repeated injection of homologous or heterologous purified glomular basement membrane into an animal. ${ }^{41}$ In either case the result is the same: antibody fixation with consequent immune injury, and, if the injections are repeated, renal failure and the death of the animal. In some animals an autonomous phase appears early in the course of the disease. This is characterized by the formation of antibody itself directed against the heterologous antibody already deposited in the kidney. ${ }^{42}$ This could provide one mechanism for initiating a chronic course in otherwise self-limiting disease. Antibodies against the damaged basement membrane itself have been sought but not found. ${ }^{43}$

Part II of this lecture will appear in next week's issue. A list of references will be given in part III, to be published in our issue of 28 October.

\section{Initial Intensive Care in an Accident and Emergency Department}

\section{R. N. BAIRD, J. NOBLE， D. MCLEAN}

British Medical fournal, 1972, 4, 90-92

\section{Summary}

The work in the resuscitation room is initial intensive care. This must be always available independent of inpatient resources. This demands investment in adequate equipment and staffing. Much of the work is medical

\section{South-Eastern Region of Scotland Hospital Board}

R. N. BAIRD, M.B., F.R.C.s., Rotating Registrar in General Surgery

J. NOBLE, M.B., F.R.C.S., formerly Rotating Registrar in General Surgery

D. MCCLEAN, M.B., F.R.C.s., formerly Rotating Registrar in General

Surgery rather than surgical and appropriate for physicians to treat. Our experience might help others to plan for the future.

\section{Introduction}

Probably a central emergency treatment area or resuscitation room will be provided in the new regional general hospitals. Such a service should be available 24 hours a day and adequately staffed. After the accident and emergency department of the Royal Infirmary of Edinburgh was modernized and reorganized in 1966 the work pattern of the resuscitation room was analysed. ${ }^{1}$ Since then peripheral hospitals, local general practitioners, and 